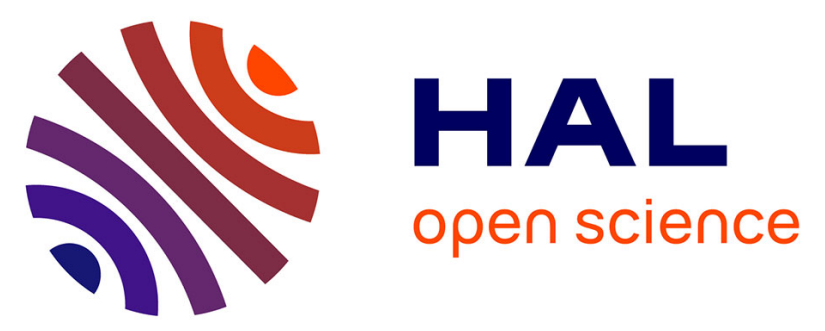

\title{
The AUTOWARE Framework and Requirements for the Cognitive Digital Automation
}

\author{
Elias Molina, Oscar Lazaro, Miguel Sepulcre, Javier Gozalvez, Andrea \\ Passarella, Theofanis P. Raptis, Aleš Ude, Bojan Nemec, Martijn Rooker, \\ Franziska Kirstein, et al.
}

\section{To cite this version:}

Elias Molina, Oscar Lazaro, Miguel Sepulcre, Javier Gozalvez, Andrea Passarella, et al.. The AUTOWARE Framework and Requirements for the Cognitive Digital Automation. 18th Working Conference on Virtual Enterprises (PROVE), Sep 2017, Vicenza, Italy. pp.107-117, 10.1007/978-3-319-651514_10. hal-01674893

\section{HAL Id: hal-01674893 \\ https://hal.inria.fr/hal-01674893}

Submitted on 3 Jan 2018

HAL is a multi-disciplinary open access archive for the deposit and dissemination of scientific research documents, whether they are published or not. The documents may come from teaching and research institutions in France or abroad, or from public or private research centers.
L'archive ouverte pluridisciplinaire HAL, est destinée au dépôt et à la diffusion de documents scientifiques de niveau recherche, publiés ou non, émanant des établissements d'enseignement et de recherche français ou étrangers, des laboratoires publics ou privés. 


\title{
The AUTOWARE Framework and Requirements for the Cognitive Digital Automation
}

\author{
Elias Molina ${ }^{1}$, Oscar Lazaro ${ }^{1}$, , Miguel Sepulcre ${ }^{2}$, Javier Gozalvez ${ }^{2}$, Andrea \\ Passarella ${ }^{3}$, Theofanis P. Raptis ${ }^{3}$, Aleš Ude ${ }^{4}$, Bojan Nemec ${ }^{4}$, Martijn Rooker ${ }^{5}$, \\ Franziska Kirstein ${ }^{6}$ and Eelke Mooij ${ }^{7}$ \\ ${ }^{1}$ Innovalia Association, Bilbao Spain \{emolina, olazaro\}@innovalia.org \\ ${ }^{2}$ Universidad Miguel Hernández de Elche, Avda. Universidad s/n, 03202, Elche, Spain \\ \{j.gozalvez, msepulcre\}@umh.es \\ ${ }^{3}$ Institute of Informatics and Telematics, National Research Council, 56124, Pisa, Italy \\ \{andrea.passarella, theofanis.raptis\}@iit.cnr.it \\ ${ }^{4}$ Jožef Stefan Institute, Department of Automatics, Biocybernetics, and Robotics, Jamova 39, \\ 1000 Ljubljana, Slovenia \{ales.ude, bojan.nemec\}@ijs.si \\ ${ }^{5}$ TTTech Computertechnik, 1040 Vienna, Austria \{martijn.rooker\}@tttech.com \\ ${ }^{6}$ Blue Ocean Robotics, Odense, Denmark $\{\mathrm{fk}\} @$ blue-ocean-robotics.com \\ ${ }^{7}$ PWR Pack, Maxwellstraat 41,6716 BX Ede, Netherlands \{Mooij\}@ pwr-pack.nl
}

\begin{abstract}
The successful introduction of flexible, reconfigurable and selfadaptive manufacturing processes relies upon evolving traditional automation ISA-95 automation solutions to adopt innovative automation pyramids. These new approaches target the integration of data-intensive cloud and fog-based edge computing and communication digital manufacturing processes from the shop-floor to the factory to the cloud. The integration and operation of the required ICT, automation and robotic technologies and platforms is particularly challenging for manufacturing SMEs, which account for more than $80 \%$ of manufacturing companies in Europe. This paper presents an insight into the business and operational processes, which motivate the development of a digital cognitive automation framework for collaborative robotics and modular manufacturing systems particularly tailored to SME operations and needs; i.e. the AUTOWARE Operative System. To meet the requirements of both large and small firms this paper elaborates on the smart integration of wellestablished SME friendly digital frameworks such as the ROS supported robotic Reconcell framework, the FIWARE-enabled BEinCPPS Cyber Physical Production framework and the OpenFog compliant TAPPS hardware framework.
\end{abstract}

Keywords: Collaborative robotics; Cyber-Physical Systems; Modular manufacturing systems; Requirements engineering; Smart Factory.

\section{Introduction and Background}

Manufacturing is the second most important sector in terms of small and mediumsized enterprises' (SMEs) employment and value added in Europe [1]. Over $80 \%$ of the total number of manufacturing companies is constituted by SMEs, which represent $59 \%$ of total employment in this sector. In a global competition arena, 
companies need to respond quickly and economically feasible to the market requirements. In terms of market trends, a growing product variety and mass customization are leading to demand-driven approaches. It is, therefore, important that production plants can handle small lot sizes and are able to quickly apply changes in product design. In this regard, optimization of manufacturing operations is a major objective for industry. Yet, it still seems difficult for SMEs to understand the driving forces behind digitalization and how they can make use of the vast variety of individualized products and solutions to digitize their manufacturing process, making them cognitive and smart. Moreover, as SMEs intend to adopt data-intensive collaborative robotics and modular manufacturing systems, making their advanced manufacturing processes more competitive, they face the problem to seamlessly connect their physical automation processes with their digital counter parts, leading to difficult and costly digital platform adoption.

This paper is organized as follows. Section 2 reviews the state of the art of the main building blocks behind cognitive digital automation processes. Next, the requirements towards cognitive digital automation are discussed in Section 3; along with the details about the AUTOWARE framework. Finally, Section 4 concludes the paper.

\section{State of the Art}

The aim of this Section is to revise the state of the art in terms of both industry 4.0 reference architectures and the five pillars that support the development of cognitive digital manufacturing systems: (1) reconfigurable collaborative robotised capabilities, (2) resilient time sensitive (wireless) communications and data distribution, (3) extension of automation equipment for app-ized operation (open trusted platforms), (4) open service development for added value cognitive industrial services and (5) cross-layer security and certification.

\subsection{Industry 4.0 Reference Architectures}

With regards to the interoperability of automation systems, the ANSI/ISA-95 and, later, the IEC 62264 standards define a hierarchical model that has been largely used as a reference for manufacturing systems, as well as for specifying interfaces to connect enterprise systems and control operations. Several standards are upon the ANSI/ISA-95, such as the ISO 15746, which is focused on the integration of advanced process control and optimization capabilities for manufacturing systems. However, instead of hierarchical architectures, the industry is moving toward flexible structures, where functions are distributed throughout multiple IT networks and interact across different control levels, as promoted by PathFinder and ScorpiuS projects. As a representative example, the Reference Architecture Model Industrie 4.0 (RAMI 4.0) is a metamodel that integrates the production system life cycle with a functional control hierarchy, by combining different standards, such as the IEC 62264 or the IEC TS 62832 standard "for the Digital Factory", which defines a framework to specify a factory using digital representation of assets. RAMI 4.0 is especially focused on the process and manufacturing industries, unlike other reference architectures, such as the Industrial Internet Consortium Reference Architecture 
(IIRA) or the IoT driven SmartM2M (ETSI), in which manufacturing is just one of the applicable sector (a vertical domain). A thorough review of current manufacturing standards is given in [2], which states that "existing manufacturing standards are far from being sufficient for the service-oriented smart manufacturing ecosystem". Emerging technologies upon which future smart manufacturing systems will rely is described below.

\subsection{Cognitive Digital Automation Enabling Platforms}

By definition of the U.S. Department of Energy's Advanced Manufacturing Office, "Smart Manufacturing is a network data-driven process that combines innovative automation and advanced sensing and control. Cognitive manufacturing integrates manufacturing intelligence in real-time across an entire production operation while minimizing energy, material use, and costs" [3]. In contrast to traditional automation, in which work cells are generally isolated from each other and based on a static production schedule, advanced manufacturing aims to optimize the production processes and schedules by adopting flexible configurations. With that goal in mind, new monitoring, coordination and communication functions are being increasingly integrated into modern production control systems. "Cognitive Factory" term appeared for the first time in 2009 [4], where the authors identified complex adaptive systems to react autonomously and flexibly to changes. Thus, a Cognitive Factory performs adaptive responses by continuously extracting information and properties from physical objects (e.g., machines, work pieces, etc.) and production processes. Furthermore, besides implementing machine learning and predictive management capabilities, smart factories must rely on reliable networks both at the shop-floor and the enterprise-wide level, and on robots that autonomously adapt to dynamic environmental changes.

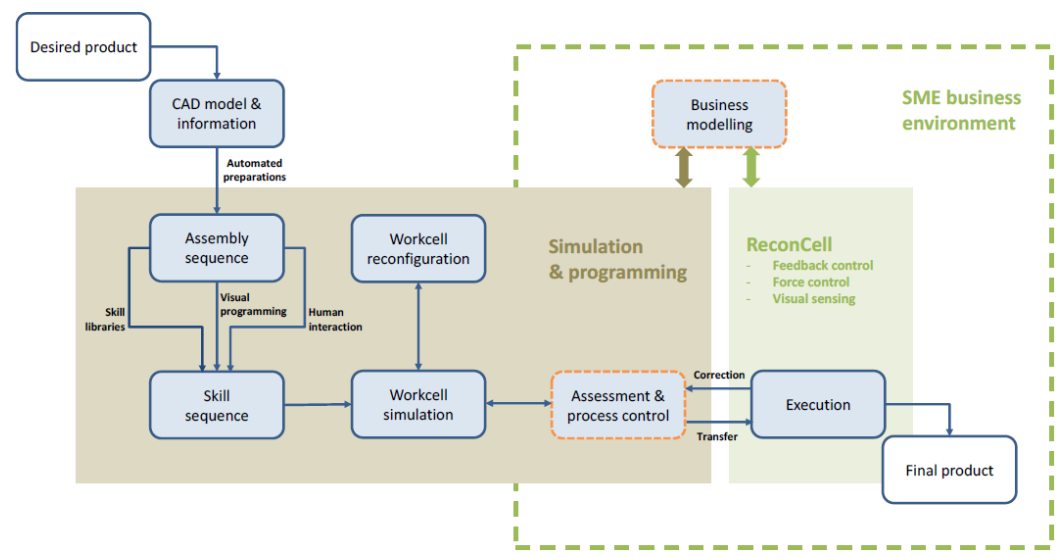

Figure 1. Block diagram sketches ReconCell workflow for new product preparation. 


\subsubsection{Reconfigurable Work Cells}

Flexibility of individual work cells has been a research topic for some years [5]. Moreover, the ability of fast reconfiguration of the work cell [6] turns to be even more important. Even more attractive is the possibility of partial or total automatic reconfiguration of the work cells. In such case, robots do not only play the role of universal manufacturing tools, but they are able of automatically reconfiguring the entire work cell layout, including their own base position. This challenge is addressed, for example, in the ReconCell project (http://www.reconcell.eu/), which aims to propose low cost, flexible integrated software and hardware production platform, which will enable quick setup and automatic reconfiguration (see Figure 1). It incorporates state of the art technologies, such as programming-by-demonstration, adaptive force control, accurate 3D vision sensors, reconfigurable passive elements, intuitive visual programming supported by simulation, use of interchangeable agents based on plug-and-produce approach and optimal gripper design supported by $3 \mathrm{D}$ printing technology.

\subsubsection{Resilient industrial wired and wireless communication networks}

Until now, no real-time communication has been supported in IEEE standardized Ethernet, which caused the emergence of proprietary modifications for industrial automation. To overcome interoperability problems, the IEEE Time-Sensitive Networking (TSN) task group is standardizing real-time functionality in Ethernet. Moreover, OPC UA (Open Platform Communication Unified Architecture) has been identified as the reference standard for Industry 4.0. Besides, the Publish/Subscribe enhancement for OPC UA allows for multicast transmission between sensors, machines and the cloud, which combined with IEEE TSN makes the vision of open, real-time machine to machine communication a reality for multi-vendor applications. In addition, industrial wireless networks play a key role in the cost and time reduction for deployment of plug-and-produce systems, enabling the connectivity of mobile systems and robots. However, mission-critical applications, demand reliable and low-latency communications. WirelessHART and ISA100.11a are the most common wireless standards for automation and control systems. They are based on the IEEE 802.15.4 physical and Medium Access Layers, use Time Division Multiple Access (TDMA) combined with Frequency Hopping, and both implement a centralized management architecture. WirelessHART reliability, latency or efficiency improvements include multipath routing protocols, link scheduling schemes or relaying and packet aggregation [7]. Furthermore, the interest of IEEE 802.11 in industrial environments started with 802.11e, which allowed prioritization by using the Enhanced Distributed Channel Access (EDCA). 802.11 network reliability enhancements propose the tuning of novel rate adaptation algorithms, and the deactivation of carrier sensing and backoff procedures. More recently, wireless seamless redundancy has been proposed in [8], relying on the IEC 62439-3 Parallel Redundancy Protocol (PRP).

Finally, 4G cellular networks (based on LTE, Long Term Evolution), initially intended for mobile broadband applications, were not suitable for strict and deterministic industrial QoS requirements. However, factory automation is now a key 
objective for beyond LTE and 5G networks (3GPP Release 13 onwards); e.g., overprovisioning through instant uplink access (IUA) [9]. Proposals to improve the reliability of cellular transmissions generally focus on the use of spatial and frequency diversity; with novel multi-hop routing diversity being proposed.

\subsubsection{Data Management and Elaboration in Large-scale Industrial Networks}

Data-centric operations and decentralization are two fundamental cornerstones of modern industrial automation technologies and form the basis for decision making and control operations [10]. In this context, cloud computing technologies can be used to implement different types of data-centric automation services at reduced costs [11]. However, deploying control-related services in clouds poses significant challenges. For example, jitters become more problematic, as well as a possible loss of control over the data by the legitimate owners. Therefore, it is widely recognized that centralized solutions to collect data in industrial environments are not adequate. The fact that automation control may span multiple physical locations and include heterogeneous data sources, and the upcoming Internet of Things (IoT) make decentralized data management inevitable. This is one of the aspects where Fog Computing, placed between the cloud and the actual machines or plant, can come into play. This will enable more efficient processing, analysis and storing of the data, thereby reducing the delay in communication between the cloud and the machines and providing opportunities for latency-sensitive applications. Components that realize the fog computing architecture are called fog nodes and are characterized by their nonfunctional properties like e.g., real-time behavior, reliability, availability, safety and security.

\subsubsection{Open Cognitive Industrial Service Development Platforms}

The Future Internet (FI) PPP has designed a FI core ICT platform, FIWARE, which is released to the general public as an open source project through the FIWARE Foundation. FIWARE enables software engineers and service providers to develop innovative digital products and infrastructures in a vendor-neutral and cost-effective way. It delivers not only a very rich set of open APIs (i.e., Generic Enablers targeted at a wide range of applications), but a catalogue of open source reference implementations. In fact, many of the current digital manufacturing platforms being developed in Europe [12]; e.g., BEinCPPS, PSYMBIOSYS, NIMBLE, vf-OS, among other projects, are adopting $\mathrm{F}$ enablers. As a representative example, the BEinCPPS project applied this paradigm to the manufacturing industry, and it provides a smart manufacturing service development framework that support data exploitation from the shop-floor to the factory to the cloud connecting the 3 domains of the Factory 4.0; i.e. the Smart Factories (Internet-of-Things, IoT), the Digital Factories (Internet-ofServices, IoS) and the Virtual Factories (Internet-of-People, IoP). BEinCPPS provides a RAMI 4.0 compliant blueprint as an integrated bundle of FIWARE Generic Enablers (GE) and Industry 4.0 Specific Enablers (SE) developed in projects such as CREMA, C2NET and FITMAN. FIWARE technologies and open source enablers 
and implementations provide basic support for typical usage scenarios in their target domain, and can be further customized to fit additional needs.

\subsubsection{Cross-layer Security and Certification}

With the convergence of Operational Technology (OT) and Information Technology (IT) systems, manufacturers raise concerns about security and confidentiality risks because data is now exchanged between multiple networks. In this regard, ISO/IEC 2700X standards provide a set of guidelines to perform IT protection, including different techniques to prevent, detect and manage cyber-attacks. For example, cryptography modules, firewalls and access control lists, intrusion prevention/detection systems, antivirus, or Security Information and Event Management (SIEM) software. A sector-specific perspective can be found in the ISA/IEC 62443 standard, which defines procedures to implement secure automation and control systems. Needless to say, to protect manufacturing lines, it is important to secure machine-to-machine (M2M) communications by using secure protocols or tunneling schemes, and to enforce integrity and authenticity of sensor data. From the counterpart's perspective, the cryptographic services shall not significantly degrade the performance or availability of industrial control services designed to operate without cryptographic protection.

Regarding certification-related aspects, they are a priority in manufacturing scenarios. Certifying safety and security is simplified by the use of standard-based procedures. ISO 10218-X series are the most relevant safety standards for applications of industrial robots. This series are supplemented by the ISO/TS 15066, which specifies safety requirements for collaborative industrial robot systems and the work environment. From a software point of view, for example, the ISO/IEC 15408 standard is commonly used in software certifications; and the ISO/IEC 25010 is focused on the software quality, dealing with aspects of preserving access to data and their modification.

\section{AUTOWARE: A Cognitive Digital Operating System}

As discussed above, the number of technologies to be integrated to realize a cognitive automation system is large. AUTOWARE (www.autoware-eu.org) proposes an architecture (Figure 2) based on intensive piloting and solid technological foundations for development of cognitive manufacturing in autonomous and collaborative robotics as extension of ROS/Reconcell frameworks, and for modular manufacturing solutions based on RAMI 4.0. AUTOWARE acts as an Open Digital Automation Operating System (OS) providing full support for digital automation from shop-floor to the cloud.

\subsection{AUTOWARE Cognitive Automation Reference Architecture}

To facilitate a shift from product-centric to user-centric business models, the layered architecture proposed by the Smart Service Welt initiative defines [13] "smart spaces" where Internet-enabled machines connect to each other, and "smart products" that 
also encompasses their virtual representations (CPS digital twins). These products know their own manufacturing and usage history and are able to act autonomously. In this architecture, data generated on the networked physical objects are consolidated and processed (smart data) on software-defined platforms, and providers connect to each other via these service platforms to form digital ecosystems. AUTOWARE extends those elements that are critical for the implementation of the autonomy and cognitive features. Specifically, AUTOWARE leverages enablers for deterministic wireless CPPS connectivity (TSN-OPC UA and Fog-enabled analytics) at the smart product level. At the smart data level, the approach is to develop cognitive planning and control capabilities supported by cloud services and dedicated data management systems, which will contribute to meet the real-time visibility and timing constrains of the planning and control algorithms for autonomous production services. At the smart service level, AUTOWARE helps to model and secure trusted CPPS, and their self-configuration policies. In this latter aspect, the incorporation of the TAPPS CPS framework coupled with the provision of a smart automation service store, will pave the way towards an open service market for digital automation solutions cognitive bydesign. AUTOWARE cognitive OS makes use of a combination of reliable M2M communications, human-robotics-interaction, modelling \& simulation, and cloud-fog based analytics schemes. In addition, considering the mission-critical requirements, this combination is deployed in a secure and safe environment. All this should enable a reconfigurable manufacturing system that enhances business productivity.

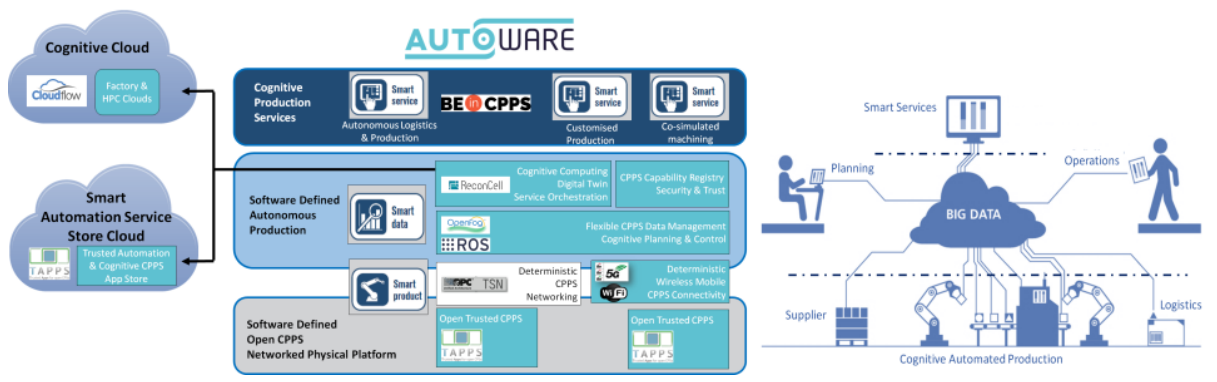

Figure 2. AUTOWARE Reference Architecture for Cognitive Manufacturing.

\subsection{Cognitive Automation Manufacturing Process Requirements and Extensions}

The implementation of advanced cognitive manufacturing processes poses challenges in terms of how data should be communicated (latency, reliability) and distributed (availability), and how to react to the working environment (reconfigurability). The following presents two use cases (cognitive-packaging, human-robot collaboration) and associated requirements that motivate the advanced features provided by AUTOWARE at communication, data distribution and service adaptation level. 


\subsubsection{Smart Data Management Requirements and AUTOWARE Extensions}

Regarding the communication requirements of industrial automation. ETSI divides [14] automation systems into three classes (manufacturing cell, factory hall, and plant level) with different needs in terms of latency $(5 \mathrm{~ms} \pm 10 \%, 20 \mathrm{~ms} \pm 10 \%$, and $20 \mathrm{~ms} \pm 10 \%)$ and update time $(50 \mathrm{~ms} \pm 10 \%, 200 \mathrm{~ms} \pm 10 \%$, and $500 \mathrm{~ms} \pm 10 \%)$. However, all three classes require a 10-9 packet loss rate and a 99.999\% application availability. These requirements are confirmed by PWR Pack AUTOWARE use case; a company that develops robotic systems for loading individual products into packaging machines or finished packs into shipping containers. PWR Pack is working on reducing the machine recipe change-over times, and enhance the cognitive and reconfigurability of a packaging line so that it can handle different packaging formats and products with adaptive and auto learning calibration. To this aim, PWR Pack relies on sensor fusion, flexible real-time data processing with programmable hardware and model based analysis and control. Figure 3 illustrates PWR Pack's industrial validation use case that contains four main nodes. The MS (Machine System) handles the product distribution. The HMI (Human Machine Interface) is used by the operators for a day-to-day control using 3D visualization. The PLC (Programmable Logic Controller) controls the servomotors at robots, conveyors and others, and performs motion tasks and path planning for the robots based on commands received from the MS.

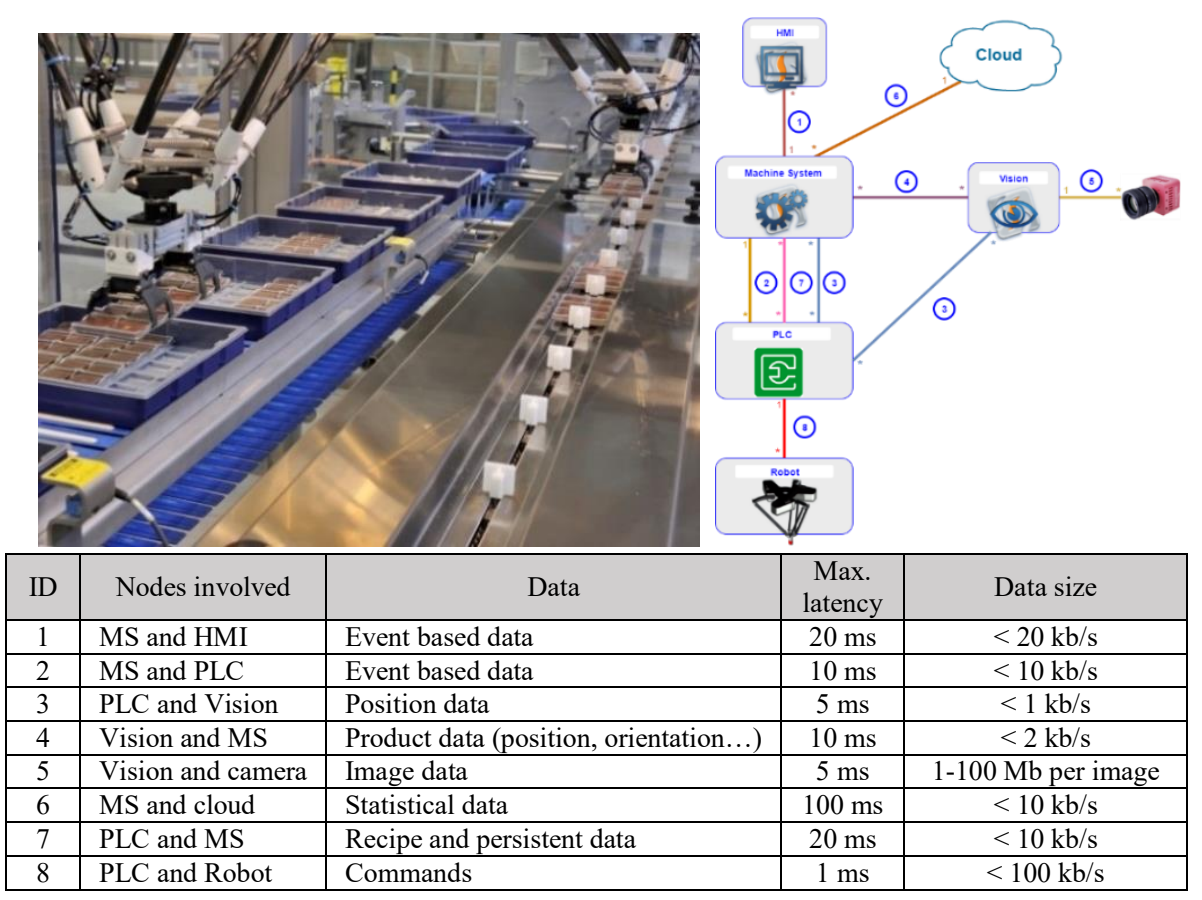

Figure 3. Requirements of the industrial cognitive validation use case by PWR Pack. 
AUTOWARE extensions focus on the management of large amounts of data through smart distribution policies. These policies are based on decentralized cognitive heuristic techniques that replicate data to locations from where they can be accessed when needed within appropriate deadlines. AUTOWARE also provides a data management process that interacts with the routing communication layer (smart product cross-layer approach) to provide efficient data access and end-to-end delay guarantees.

Furthermore, AUTOWARE software-defined autonomous production layer selectively moves data to different network areas and will devise methods on how the data requests will be served, given an underlying routing protocol. The data management module will replicate/store/move data between (i) (mobile) nodes in the factory environment (e.g., mobile nodes of operators, nodes installed in work cells, nodes attached to mobile robots, etc.) (ii) edge nodes providing storage services, and (iii) remote cloud storage services. All the three layers will be used in a synergic way, based on the properties of the data and the requirements of the users requesting it.

\subsubsection{AUTOWARE Smart Product Autonomous Adaptation Service Extensions}

A truly flexible and reconfigurable work cell should not rely on the specific hardware solution. Moreover, it has to enable quick and transparent change of the specific hardware without applying new programming tools. Thus, the work cell as a basic entity of the flexible production should be unified around the common software architecture. A suitable framework for such an architecture is offered by the Robot Operating System (ROS), which provides tools to create platform independent applications. ROS nodes (i.e., software modules that typically controls a robot, handles vision system, runs simulation system or heavy computing of motion planning, etc.) can communicate directly to each other by passing messages and creating services. AUTOWARE provides an extension (Figure 4) to the ReconCell software architecture in the form of abstraction to support different robots. The aim is to apply a number of trajectory and feedback control strategies independently of the selected robot, and enable the programming of new strategies via a suitable control interface. For this reason, a real-time server accepts higher-level ROS commands and applies the control strategies to execute the desired robot motion. 


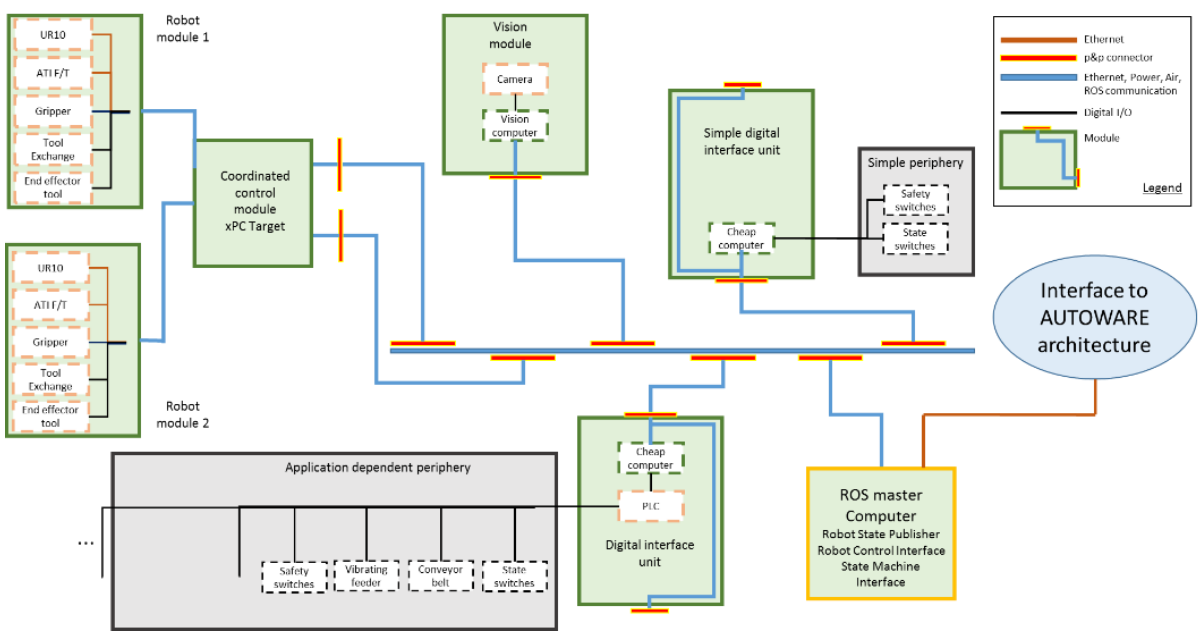

Figure 4. AUTOWARE ROS-based cognitive reference architecture.

Taking into account the described infrastructure, several enhancements (Figure 5) are provided by AUTOWARE: autonomous adaptation of robot trajectories (improve reinforcement learning and adaptation of the robot skills and policies by more focused search using previous knowledge), bimanual robot task execution (provide programming tools to facilitate learning by demonstration), self-organization and optimal configuration of the work cell layouts for the given task (simultaneously optimizing both optimal layout design via simulation and search algorithms; while control policies will apply iterative linear quadratic regulator techniques).

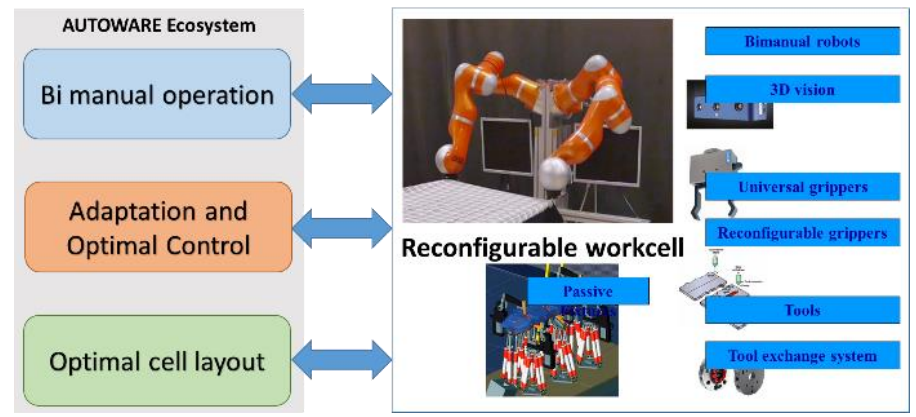

Figure 5. Enhancement of the existing reconfigurable work cell.

\section{Conclusions and Further Research}

This paper has presented the main components and reference architectures for the development of cognitive manufacturing systems. The paper has introduced AUTOWARE, a novel RAMI 4.0 open platform for fast and effective development and optimal operation of cognitive manufacturing services. Technical requirements from selected AUTOWARE use cases, in terms of service adaptation and smart data 
communication and distribution for such advanced manufacturing capabilities have been discussed. The extensions needed to address such requirements have also been presented. Future research will elaborate on the performance that can be expected from the digital automation enhancements towards cognitive manufacturing solutions.

Acknowledgments. This work has been funded by the European Commission through the FoF-RIA Project AUTOWARE: Wireless Autonomous, Reliable and Resilient Production Operation Architecture for Cognitive Manufacturing (No. 723909).

\section{References}

1. P. Muller, S. Devnani, J. Julius, D. Gagliardi and C. Marzocchi, "Annual Report on European SMEs 2015/2016,” European Union, 2016.

2. Y. Lu, K. C. Morris and S. Frechette, "Current standards landscape for smart manufacturing systems.," National Institute of Standards and Technology, NISTIR, 2016.

3. Advanced Manufacturing Office (AMO), U.S. Department of Energy., [Online]. Available: https://energy.gov/eere/amo/next-generation-manufacturing-processes.

4. M. F. Zaeh, M. Beetz, K. Shea, G. Reinhart, K. Bender, C. Lau, M. Ostgathe, W. Vogl, M. Wiesbeck, M. Engelhard, C. Ertelt, T. Rühr and M. Friedrich, "The Cognitive Factory," in Changeable and Reconfigurable Manufacturing Systems, London, Springer, 2009, pp. 355371.

5. M. Hedelind, "On reconfigurable robotic working cells - A case study," Manufacturing Systems and Technologies for the New Frontier, pp. 323-328, 2008.

6. I. M. Chen, "Rapid response manufacturing through a rapidly reconfigurable robotic workcell," Robotics and Computer-Integrated Manufacturing, 17(3), pp. 199-213, 2001.

7. M. Sepulcre, J. Gozalvez and B. Coll-Perales, "Multipath QoS-driven routing protocol for industrial wireless networks," Journal of Network and Computer Applications, vol. 74, pp. 121-132, 2016.

8. G. Cena, S. Scanzio and A. Valenzano, "Seamless Link-Level Redundancy to Improve Reliability of Industrial WiFi Networks," IEEE Transactions on Industrial Informatics, 12(2), pp. 608-620, 2016.

9. B. Holfeld, D. Wieruch, T. Wirth, L. Thiele, S. A. Ashraf, J. Huschke, I. Aktas and J. Ansari, "Wireless Communication for Factory Automation: an opportunity for LTE and 5G systems," IEEE Communications Magazine, 54(6), pp. 36-43, 2016.

10. S. Yin, S. X. Ding, and X. Xie, "A review on basic data-driven approaches for industrial process monitoring," IEEE Transactions on Industrial Electronics, 61(11), pp. 6414-6428, 2014.

11. T. Hegazy and M. Hefeeda, "Industrial Automation as a Cloud Service," IEEE Transactions on Parallel and Distributed Systems, 26(10), pp. 2750-2763, 2015.

12. European Factories of the Future Research Association (EFFRA), "Platforms for Connecte Factories of the Future," Brussels, Belgium, 2015.

13. H. Kagermann, F. Riemensperger, D. Hoke, J. Helbig, D. Stocksmeier, W. Wahlster and D. 
Schweer, "Smart Service Welt: Recommendations for the Strategic Initiative Web-based Services for Businesses," acatech, Berlin, 2014.

14. ETSI TR 102 889-2 V1.1.1, "Electromagnetic compatibility and Radio spectrum Matters; System Reference Document; Short Range Devices; Part 2: Technical characteristics for SRD equipment for wireless industrial applications using technologies different from Ultra-Wide Ban,” 2011. 